\title{
MOORE-PENROSE INVERSE OF CONDITIONAL TYPE OPERATORS
}

\author{
M. R. JABBARZADEH AND M. Sohrabi ChEGEni
}

Abstract. We prove some basic results on some Moore-Penrose inverse of conditional type operators on $L^{2}(\Sigma)$. For instance, we show, among other results, that a weighted conditional operator $T=M_{w} E M_{u}$ is centered if and only if $T^{\dagger}$, the Moore-Penrose inverse of $T$, is centered. In addition, we establish lower and upper bounds for the numerical range of $T$ and $T^{\dagger}$.

Mathematics subject classification (2010): 47B20, 47B38.

Keywords and phrases: Moore-Penrose inverse, Aluthge transformation, conditional expectation, polar decomposition.

\section{REFERENCES}

[1] A. Ben-Israel And T. N. E. Greville, Generalized inverses: theory and applications, Second Ed., Springer, 2003.

[2] J. Ding And W. E. Hornor, A new approach to Frobenius-Perron operators, J. Math. Anal. Appl. 187 (1994), 1047-1058.

[3] D. S. Djordjević And N. C. Dinčić, Reverse order law for the Moore-Penrose inverse, J. Math. Anal. Appl. 361 (2010) 252-261.

[4] Y. EstaRemi, M. R. JABBARZADEH, Weighted lambert type operators on Lp-spaces, Oper. Matrices 7 (2013), 101-116.

[5] Y. Estaremi, M. R. JABBARZADEH, Weighted composition Lambert-type operators on $L^{p}$ spaces, Mediterr. J. Math. 11 (2014), 955-964.

[6] Y. Estaremi, Some classes of weighted conditional type operators and their spectra, Positivity 19 (2015), 83-93.

[7] Y. ESTAREMI, Centered weighted conditional type operators, arxiv.org.

[8] T. FuRUTA, Invitation to linear operators, Taylor \& Francis, Ltd., London, 2001.

[9] J. J. GRobleR AND B. DE PAGTER, Operators representable as multiplicationconditional expectation operators, J. Oper. Theory 48 (2002), 15-40.

[10] J. HeRRON, Weighted conditional expectation operators, Oper. Matrices 5 (2011), 107-118.

[11] M. R. JabBarZADEH, A conditional expectation type operator on $L^{p}$ spaces, Oper. Matrices 4 (2010), 445-453.

[12] I. B. JUnG, E. Ko AND C. PEARCY, Aluthge transforms of operators, Integral Equations Operator Theory 37 (2000), 437-448.

[13] B. B. Morrell and P. S. Muhly, Centered operators, Studia Math. 51 (1974), 251-263.

[14] A. LAMBERT, $L^{p}$-multipliers and nested sigma-algebras, Oper. Theory Adv. Appl. 104 (1998), 147153.

[15] A. LAMBERT AND B. WeINSTOCK, A class of operator algebras induced by probabilistic conditional expectations, Michigan Math. J. 40 (1993), 359-376.

[16] M. M. RAO, Conditional measure and applications, Marcel Dekker, New York, 1993. 